\title{
Le decisioni manageriali e la rivoluzione comportamentale*
}

\author{
MARIA LAURA FRIGOTTO ${ }^{* *}$ ALESSANDRO ROSSI ${ }^{* \star}$ \\ SANDRO TRENTO ${ }^{* * *}$ ENRICO ZANINOTTO ${ }^{* * * *}$
}

\begin{abstract}
Obiettivo del paper: Il paper descrive come economia sperimentale e psicologia cognitiva abbiano influenzato lo sviluppo degli studi comportamentali sulle decisioni all'interno delle discipline manageriali; critica l'approccio comportamentale largamente dominante negli studi di management; propone di recuperare l'eredità della c.d. scuola di Carnegie come elemento fondativo di una piena rivoluzione comportamentale nello studio delle decisioni manageriali.

Metodologia: Il lavoro adotta una prospettiva comparativa e interdisciplinare per discutere l'applicazione dell'approccio comportamentale allo studio delle decisioni nelle discipline e nella pratica manageriale.

Risultati: Per un pieno compimento della "rivoluzione comportamentale" negli studi di management, il paper indica la necessità di integrare l'approccio basato sulla correzione del bias decisionale utilizzando le evidenze comportamentali in chiave evolutiva, ecologica e costruttivista.

Limiti della ricerca: Il presente lavoro non affronta tutta la letteratura sul processo decisionale manageriale, ma si limita al confronto fra recenti studi sperimentali e l'approccio comportamentale della Scuola di Carnegie, per evidenziare carenze e potenzialità per lo studio teorico e applicato delle decisioni manageriali.

Implicazioni pratiche: Il paper suggerisce come una considerazione più completa degli aspetti comportamentali implichi la ridefinizione di molte pratiche decisionali del management.

Originalità del paper: Il lavoro delinea alcune linee guida per lo sviluppo di un programma di ricerca comportamentale di carattere originale per le discipline manageriali.

Gli autori desiderano ringraziare i due referee anonimi per gli utili commenti e suggerimenti ricevuti.

** $\quad$ Ricercatore in Organizzazione Aziendale e Risorse Umane - Università di Trento

e-mail: marialaura.frigotto@unitn.it

*** Professore aggregato di Economia e Gestione delle Imprese - Università di Trento e-mail: alessandro.rossi@unitn.it

**** Ordinario di Economia e Gestione delle Imprese - Università di Trento e-mail: sandro.trento@unitn.it

***** Ordinario di Economia e Gestione delle Imprese - Università di Trento e-mail: enrico.zaninotto@unitn.it
\end{abstract}


Esso integra l'uso di procedure correttive per i modelli razionali di scelta, per tenere conto di apprendimento, selezione, aspetti ecologici all'interno dei comportamenti decisionali, e del rapporto tra modelli decisionali adottati e la natura contingente dei problemi di scelta incontrati dai decisori.

Parole chiave: decisioni manageriali; comportamento decisionale; problem-solving; euristiche ed errori decisionali

Purpose of the paper: This paper describes how experimental economics and cognitive psychology have affected the managerial literature on behavioural decision-making; criticizes the widely adopted approach in the managerial literature which rely exclusively on topics and methods of modern behavioural economics; proposes a different approach, that integrates the "Carnegie tradition" as a key element for a complete behavioural revolution in managerial decision-making.

Methodology: A comparative and interdisciplinary perspective is adopted to discuss behavioural decision-making applications to management studies and practices.

Findings: To accomplish a "behavioural revolution" in management, the paper advocates the need to integrate the "decision bias correction" approach within a larger frame encompassing behavioural results in an evolutionary, ecological and constructive problem solving setting.

Research Limitations: This paper does not address the whole literature on managerial decision making, but compares recent experimental studies with the behavioural approach of the Carnegie School, aiming at highlighting shortcoming and potentiality for managerial decision making research and practice.

Practical Implications: The paper suggests that a stronger consideration of the behavioural approach to the study of decision-making would imply a revision of managerial practice.

Originality of the paper: The work provides guidelines for the development of an original behavioural research agenda integrating the study of corrections to rational choice models with the analysis of actual decision-making. This implies taking into account phenomena such as learning and selection, including the ecological side of behaviour, and to deepen the relationship between adopted decision-making models and the contingent nature of choice problems faced by decision makers.

Key words: managerial decision-making; behavioural decision-making; problem-solving; bias and heuristics

\section{Introduzione}

Si provi a inserire "decision making" come chiave di ricerca in Amazon.com. Appariranno alcune pagine di proposte editoriali. La prima ci promette di migliorare le nostre capacità decisionali: «Not only will readers learn how to make decisions, they will learn how to make the smartest decisions» ${ }^{1}$; la seconda ci sollecita

\footnotetext{
Enfasi in corsivo nell'originale.
} 
all'acquisto mostrandoci «how to overcome your brain's natural shortcomings»; viene poi un testo che ci lusinga con una «cutting-edge exploration of the mysteries of rational thought, decision-making, intuition, morality, willpower, problemsolving, prediction, forecasting, unconscious behavior, and beyond»; e ancora un libro che «presents original ideas by today's leading psychologists, neuroscientists, and philosophers who are radically expanding our understanding of human thought». La lista potrebbe continuare a lungo, ma basta il richiamo a questi bestseller per avere una dimensione di quanto le scoperte della psicologia, dell'economia sperimentale e delle neuroscienze abbiano inciso sul modo di affrontare il problema decisionale dei manager.

Tali ricerche, ora rese popolari da molti libri di successo, hanno definito uno spartiacque fondamentale che ha spostato l'attenzione da analisi di tipo formale e assiomatico del comportamento di scelta a metodi empirici di rilevazione degli effettivi comportamenti osservabili in contesti decisionali concreti. Per quanto non manchino esempi pionieristici di un approccio di taglio comportamentale nella letteratura manageriale ${ }^{2}$, è dalla "rivoluzione" avvenuta negli studi di psicologia della decisione, nell'economia sperimentale e nelle neuroscienze che i nuovi approcci alla decisione manageriale traggono la propria linfa. Questo articolo offre alcune riflessioni sull'effetto che tale rivoluzione comportamentale ha avuto e continua tuttora ad avere all'interno della tradizione di studio interessata al tema delle decisioni manageriali. La nostra tesi è che gli importanti risultati ottenuti in psicologia e in economia a seguito dell'introduzione di metodi sperimentali per lo studio del comportamento decisionale siano stati incorporati nel dibattito teorico e nella pratica manageriale alla stregua di un paradigma dominante e senza la capacità di integrare tali considerazioni con quelle provenienti da programmi di ricerca comportamentali adottati in precedenza negli studi manageriali e organizzativi.

Gli studi di economia comportamentale originano da una critica radicale dell'applicabilità dei fondamenti assiomatici della teoria economica della scelta. I risultati dell'economia comportamentale suggeriscono una serie di correttivi delle procedure decisionali tradizionali, ancorate agli schemi della decisione razionale. La prospettiva dominante è quella delle "deviazioni" dalla razionalità: si mantiene l'idea che esista un modello decisionale ottimale, ma si ammette che il suo impiego possa essere viziato da scorciatoie (euristiche decisionali) generalmente responsabili di una molteplicità di errori (bias).

La nostra posizione è che, viceversa, errori e scorciatoie decisionali rispetto al modello ideale rivelino talora una intelligente capacità di muoversi in contesti decisionali complessi. In questa luce, contributi recenti degli studi sul comportamento decisionale assumono un potenziale molto più ampio, che richiede di rifondare gli stessi studi manageriali al fine di produrre modelli e procedure

2 Si pensi al celeberrimo "effetto Hawthorne" individuato negli studi organizzativi di Mayo (1933, 1945) oppure agli studi degli anni Trenta sulle pratiche di mark up pricing (Hall e Hitch, 1939; Harrod, 1939). 
decisionali più efficienti e coerenti con la natura della cognizione umana, individuale e organizzata.

Questa prospettiva allargata dello studio analitico del comportamento umano come fondamento delle scienze della decisione ha proprio all'interno del management e della scienza dell'amministrazione precedenti illustri: basti pensare alla scuola di Carnegie (Hosseini, 2003). Questa più antica tradizione comportamentale, che chiameremo per comodità la "prima rivoluzione comportamentale", parte dal presupposto che un approccio osservativo diretto sia necessario per scoprire le procedure decisionali più efficaci attuate da agenti con limiti cognitivi, o da organizzazioni complesse in cui la conoscenza è diffusa e la capacità di reperimento e di elaborazione delle informazioni sono limitate. $\mathrm{Al}$ contrario, la "seconda rivoluzione comportamentale", quella che è diventata la tradizione dominante in economia comportamentale, tende a riproporre un modello decisionale razionale, fondamentalmente astratto, a cui tendere in maniera ideale, e a cui eventualmente imporre correttivi per evitare che i comportamenti cognitivi ripetutamente osservati determinino deviazioni rispetto all'ideale secondo una prospettiva che Bell et al., (1988) hanno definito "prescrittiva".

In questo articolo riflettiamo sulle conseguenze nelle discipline manageriali del recupero di un approccio comportamentale ispirato al programma di ricerca della scuola di Carnegie. Essi si sostanziano nel riconoscimento del ruolo centrale della complessità nei processi di governo dell'impresa e dell'interconnessione esistente fra natura del problema e strategie comportamentali per la risoluzione dello stesso (Baccarani, 2010). Nel recente dibattito sulla possibilità di definire come vere scienze le discipline manageriali (Brunetti, 2010), l'articolo muove dalle critiche all'idea di management come un insieme di ricette normative ispirate a un modello di decisione ideale e validate nel passato spostando l'attenzione a quali processi comportamentali permettano l'esecuzione di azioni intelligenti all'interno di contesti complessi e caratterizzati da condizioni di novità.

L'articolo è così organizzato: nel paragrafo 2 si presenta l'evoluzione degli studi comportamentali delineando i caratteri delle due rivoluzioni comportamentali. Nel paragrafo 3 si discute di come le scoperte recenti dell'economia comportamentale (appartenenti alla seconda rivoluzione comportamentale) siano state applicate al management e si propone un approccio alternativo allo studio delle decisioni manageriali che recupera alcune intuizioni e il metodo della prima rivoluzione comportamentale. Nel paragrafo 4 si riflette sulle conseguenze di tale cambio di paradigma all'interno delle discipline manageriali.

\section{Approccio comportamentale: significato e origini}

\subsection{La prima rivoluzione comportamentale}

Parlare di economia e management comportamentale, appare, per certi versi, ridondante. Come faceva notare lo stesso Simon (1987) nel definire la voce 
"behavioral economics" in The New Palgrave Dictionary of Economics: «Since economics is certainly concerned with human behaviour - with, as Marshall put it, '[the] study of mankind in the ordinary business of life' - the phrase 'behavioural economics' appears to be a pleonasm.» Nondimeno, l'evoluzione del senso e l'uso del termine "comportamentale" segnalano la progressiva introduzione nel modello dominante di decisione economica, basato su un impianto descrittivo di taglio assiomatico, di elementi di psicologia osservativa. Questo passaggio si è avvalso del contributo fondamentale di studiosi provenienti dalle scienze cognitive che hanno introdotto metodi e procedure di analisi empirica per lo studio delle decisioni economiche.

L'assegnazione nel 2002 del premio Nobel a Daniel Kahneman e Vernon Smith ha sancito il riconoscimento di una innovazione metodologica fondamentale, quale la legittimità del porre in discussione il fondamento assiomatico della scelta economica. La rivoluzione comportamentale in economia ha tuttavia un'origine più antica, nel secondo dopoguerra: essa non ha un ambito limitato alle scienze economiche, bensì deriva dall'entusiasmo per una più generale rifondazione delle scienze sociali come area interdisciplinare a cavallo tra scienze politiche, ed economiche, psicologia e sociologia (Augier e March, 2011). Herbert Simon con Administrative behavior: A study of decision-making processes in administrative organization (1947) e, fra gli altri, James March, Richard Cyert, William Starbuck, Oliver Williamson (la c.d. Scuola di Carnegie 1949-1960) furono, rispettivamente, iniziatore e contributori prolifici di tale movimento.

La rivoluzione comportamentale sancita da questi studiosi (e che indichiamo come la prima rivoluzione comportamentale) si fonda su due elementi: (i) le decisioni quale fondamento comune di ogni organizzazione e di ogni impresa; (ii) il metodo scientifico e l'osservazione del comportamento come base empirica per qualsiasi teoria sulle organizzazioni o sulle imprese. L'apporto innovativo dei contributi della prima rivoluzione comportamentale all'economia non è tanto nell'oggetto di studio, le decisioni economiche, quanto nell'approccio adottato per studiarle, fondato su osservabilità e testabilità. Tradizionalmente invece, l'economia era e in parte è ancora basata su una psicologia elementare assiomatizzata che non si fonda sull'osservazione di comportamenti effettivi. Negli studi classici in economia, la scelta è modellizzata come l'esito di un calcolo razionale inscritto nelle premesse del problema (in cui sono dati fini, mezzi e preferenze) e che «prescinde dai processi mentali dei singoli individui» (Egidi, 2005). Le condizioni interne al decisore, ossia il modo con cui le informazioni sulle alternative sono elaborate, restano viceversa escluse dall'analisi: gli individui sono assiomaticamente descritti attraverso la definizione dello scopo del loro lavoro, ossia la realizzazione dei propri obiettivi, e non attraverso il processo con cui lo realizzano. L'economia indica la scelta che un individuo razionale opererebbe e si fonda sull'assunto implicito che gli individui in media si comportino in modo razionale come la teoria ipotizza.

La prima rivoluzione comportamentale mette in discussione tale assunto. Per usare ancora il linguaggio di Simon, non sempre si è in grado di specificare un sistema interno (decisore) in grado di compiere esattamente il compito prescritto. In 
realtà, come si è visto, lo si deve assumere, ma nulla assicura che tale capacità sia coerente con le caratteristiche fisiche della mente umana. Se si abbandona questa ipotesi, il comportamento osservato dipenderà in parte dal contesto in cui il decisore è chiamato a operare (task environment), in parte dai limiti delle proprietà interne al sistema relativamente alle condizioni ambientali in cui esso è posto, con conseguenze molto rilevanti sul contenuto normativo delle deduzioni che si possono trarre dall'osservazione dei comportamenti. Questo è ben chiaro a Simon quando, in The Science of the Artificial (1996), afferma:

\begin{abstract}
«if we could always specify a protean inner system that would take on exactly the shape of the task environment, designing would be synonymous with wishing (...) But the design has not been achieved until we have discovered at least one realizable inner system obeying the ordinary natural laws $[\ldots]$ With this shift, the theory of the firm becomes a theory of estimation under uncertainty and a theory of computation» (pp. 12 e 27)
\end{abstract}

Questa prospettiva chiama automaticamente in campo la psicologia, quale disciplina con oggetto lo studio empirico del comportamento umano (Simon, 1947). Alle origini, il modo adottato per studiare le decisioni effettive è quello che oggi diremmo dei "metodi misti", dove, in mancanza di evidenze definitive da parte della psicologia, su come il ragionamento avvenga effettivamente, si utilizzano una serie di metodi diversi, come quello computazionale, che genera evidenza empirica sul comportamento di scelta basandosi su poche intuizioni derivate dall'esperienza di scelta personale (Simon, 1955) o suggerite da studi in altri ambiti quali la biologia o la stessa psicologia (March e Simon, 1993). L'osservazione della decisione porta automaticamente anche alla considerazione che la scelta avviene, in contesti organizzativi, secondo "calcoli" che non necessariamente razionalizzano le risorse e dove il processo di decisione ha un impatto sulla scelta stessa (Cyert e March, 1963).

In questo contesto, il messaggio della prima rivoluzione comportamentale trova un più proficuo accoglimento nell'ambito degli studi organizzativi, dove vengono proposti modelli diversi da quelli della scelta razionale, come il modello dell'appropriatezza (March, 1994), il modello del cestino di rifiuti (Cohen et al.,1972) e il modello della coalizione dominante (Cyert e March, 1963). Al contrario, gli studi di economia e management proseguono a investigare e diffondere la loro base esperienziale e tecnica mantenendo la teoria della decisione razionale come riferimento.

\title{
2.2 La seconda rivoluzione comportamentale
}

Più recentemente, è possibile individuare una seconda rivoluzione comportamentale, la cui rilevanza è stata riconosciuta con l'assegnazione dei premi Nobel di cui si è detto in precedenza. Essa prende le mosse da una serie di evidenze secondo cui «l'azione ed il pensiero umani sono caratterizzati da sistematiche 
'deviazioni' da ciò che le teorie economiche standard considerano, rispettivamente, l'agire razionale e il ragionamento logico» (Egidi, 2005).

Non c'è una visione interamente condivisa dell'economia comportamentale, se non per la comune ricerca di un maggior realismo psicologico nella descrizione dei comportamenti economici che il più delle volte si avvale di metodi sperimentali. Il punto fondamentale è la ricerca di una maggiore comprensione del comportamento dei soggetti economici all'interno di contesti decisionali individuali o caratterizzati da forme di interazione strategica, come nel programma di ricerca della teoria dei giochi comportamentale. Dall'osservazione controllata delle decisioni dei soggetti, si viene a conoscenza dell'esistenza di sistematiche deviazioni dal comportamento ottimizzante descritto dai manuali di microeconomia. Limitandoci ai casi più conosciuti, queste possono riguardare: aspetti della funzione di preferenza non considerati nella massimizzazione dell'utilità individuale, come nel caso delle c.d. preferenze sociali (Charness e Rabin, 2002; Fehr e Camerer, 2007) e in quello dei comportamenti equi e caratterizzati da reciprocità (Fehr e Gächter, 2000; Fehr e Schmidt, 1999; Henrich et al., 2001); valutazioni non simmetriche nell'area dei guadagni o delle perdite, come nel caso della teoria del prospetto (Kahneman e Tversky, 1979), e più in generale l'influenza dei punti di riferimento iniziali nei giudizi, come nel bias dell'ancoraggio e aggiustamento (Tversky e Kahneman 1974); rovesciamento delle preferenze in funzione della modalità con cui sono fatte esprimere ai soggetti (Lindman, 1971; Slovic e Lichtenstein, 1968) o rispetto al modo con cui il problema viene presentato (come nell'effetto di incorniciamento, cfr. Tversky e Kahneman 1981), oppure ancora rispetto a caratteristiche teoricamente irrilevanti delle alternative, di cui l'effetto similarità (Tversky, 1972), l'effetto attrazione (Devetag et al.,1997; Huber et al.,1982), l'effetto compromesso (Simonson, 1989) e l'effetto dell'esca fantasma (Choplin e Hummel, 2005; Pettibone e Wedell, 2007) sono tipiche esemplificazioni ${ }^{3}$. Queste e molte altre evidenze producono due risultati. Da un lato evidenziano errori sistematici nella valutazione (bias) che possono essere corretti con adeguate procedure; dall'altro permettono di pervenire a una generalizzazione dell'economia neoclassica attraverso l'aggiunta di parametri alla teoria (tipicamente alla funzione di utilità) nella direzione di assicurare maggiore realismo psicologico alla stessa (Camerer e Loewenstein 2004) ${ }^{4}$. Secondo queste teorie, il modello economico standard rappresenta una particolare parametrizzazione del modello generalizzato.

L'aumento di potenza descrittiva che risulta dall'ondata di studi che segue i lavori pioneristici di Daniel Kahneman, Amos Tversky e Paul Slovic (per citare solo

3 Per una trattazione più completa si rinvia ai lavori di Bazerman e Moore (2009); Camerer (2003); Rossi (2013); Shapira (1997).

4 In Bell et al., (1988) i due aspetti sono evidenziati con riferimento, rispettivamente, alle procedure di valutazione dell'incertezza, per la quale si possono individuare, con un approccio prescrittivo, procedure di correzione delle incoerenze, e della valutazione dell'utilità delle scelte, per le quali occorre anche interrogarsi sulla necessità di ampliare il modello di base per includere elementi di valutazione esclusi dai modelli tradizionali. 
gli iniziatori più noti) è senza precedenti. Sarebbe difficile oggi parlare di andamenti anomali nel mercato finanziario, o interpretare le reazioni dei consumatori ad alcune offerte pubblicitarie senza tener conto di alcuni dei bias decisionali scoperti in questi anni. Pur tuttavia, è necessario rimarcare la forte differenza metodologica e teleologica che corre tra le due rivoluzioni comportamentali. Per Simon e la scuola di Carnegie, il problema è di scoprire, attraverso l'osservazione diretta, come opera la macchina decisionale di individui e organizzazioni il cui funzionamento è vincolato da una struttura fisica che ne limita i comportamenti. Si tratta, in altri termini, di scoprire empiricamente le procedure impiegate da soggetti caratterizzati da precisi limiti cognitivi per adattarsi all'ambiente in cui essi sono chiamati a operare. L'obiettivo è di individuare una teoria del comportamento decisionale empiricamente fondata e che permetta di dar conto della ricchezza delle evidenze raccolte in una molteplicità di contesti di problem solving. Con questo spirito si osservava, ad esempio, il comportamento dei giocatori di scacchi per comprendere come risolvere un problema di dimensione non trattabile, a causa dei limiti computazionali. Nella seconda rivoluzione comportamentale, viceversa, l'attenzione è rivolta a quei comportamenti "anomali" che i soggetti pongono sistematicamente in atto nel contesto di esperimenti controllati e che non sono contemplati dalle teorie standard (anche se a volte non sono esplicitamente in contrasto con esse). I risultati della seconda rivoluzione dovrebbero servire, secondo alcuni autori, a migliorare la capacità descrittiva dei modelli classici con cui si rappresentano e stilizzano le scelte.

L'aumento di capacità descrittiva e predittiva ha poi significative conseguenze nell'impiego in senso normativo e prescrittivo dei modelli, qualora si tratti di rispondere alla domanda: «come risponderanno i mercati e le istituzioni a queste caratteristiche non standard?» (Della Vigna, 2009). Ciò equivale a chiedersi che cosa farebbe un attore razionale, capace di descrivere il mondo nei termini previsti dalle teorie comportamentali. Evidentemente i decisori adatterebbero le proprie funzioni decisionali per tener conto di agenti soggetti a tutti i bias e le particolarità comportamentali descritte. Naturalmente questo significa che esistono decisori con caratteristiche non standard, ed attori che - per le loro esperienze passate, o per l'accesso a migliori conoscenze - possono attivare procedure decisionali "non viziate", tra questi vi sono le imprese: «Unlike individual consumers, firms can specialize, hire consultants, and obtain feedback from large data sets and capital markets» (Della Vigna 2009). È questa la prospettiva che Bell et al. (1988) definiscono "prescrittiva", preoccupata dalla discrepanza tra la teoria del comportamento razionale e il modo in cui effettivamente gli individui si comportano, e ispirata al desiderio di ricondurre, attraverso un po' di "psychological therapy", a un ideale normativo individui che presentano comportamenti definiti come difformi all'ideale dall'analisi descrittiva. Secondo tale ottica, il decisore "deviante" è «gently confronted with the observation that some of his responses are seemingly inconsistent and may be invited to think more deeply about these inconsistencies» (p. 23). 
Le conseguenze prescrittive della seconda rivoluzione comportamentale risultano immediate se si rappresenta, per usare l'espressione di Alvin Roth (2002), l'economista come un ingegnere. Attraverso esperimenti controllati, infatti, è possibile distinguere, nell'osservazione dei comportamenti, fra fattori legati all'ambiente in cui i soggetti operano e fattori legati agli stati interni dei decisori. L'economista a questo punto si pone come un designer esterno che usa il modello descrittivo (che comprende un modello dell'ambiente e un modello di comportamento dei decisori) per intervenire sulle variabili di controllo e produrre le condizioni desiderate. All'interno di questo quadro interpretativo, le "anomalie" comportamentali rispetto al modello di decisione razionale non costituiscono che una complicazione in più da considerare per valutare la reazione degli agenti ai cambiamenti nelle variabili controllate dal designer ${ }^{5}$.

Avremo quindi un agente razionale (l'economista ingegnere, o designer) che sfrutta una descrizione del mondo nella quale si tiene conto che il comportamento degli agenti è soggetto a una sorta di parametrizzazione allargata. Così, ad esempio, nel definire una struttura ottima di incentivo non si potrà trascurare che la reazione empiricamente osservata dei soggetti a incentivi monetari può comportare la perdita di motivazioni intrinseche (Gneezy e Rustichini, 2000), o lo spostamento rispetto all'identità sociale rilevante per il soggetto (Akerlof e Kranton, 2010).

È chiaro che in questo procedimento esiste un elemento di contraddizione: l'economista-designer è una specie di essere iper-razionale, il quale conosce le debolezze dei suoi simili, ma lui stesso ne è invece esente. I suoi simili di cui descrive il comportamento, a loro volta, "non sanno che il designer sa", che sfrutta le loro debolezze, e continuano a mettere i propri bias decisionali al servizio degli obiettivi del designer. Del pari, viene evitata la domanda sui motivi per cui gli esseri umani siano costituiti in quel modo, perché la selezione naturale non abbia premiato individui interamente razionali e abbia prodotto una specie in cui la larghissima maggioranza delle persone è indotta facilmente a valutazioni sbagliate ${ }^{6}$. Ma per la dimensione propria dell'economia queste difficoltà hanno un'importanza relativa: se i decisori sui quali si interviene sono una molteplicità di attori atomistici, si può ben pensare che i comportamenti decisionali siano abbastanza stabili, non risentano di quanto conosce l'esperto, e si possano pertanto progettare modelli di matching, strutture di remunerazione, istituzioni capaci di produrre beni pubblici che sfruttano adeguatamente le nuove conoscenze dell'economia comportamentale.

5 In una direzione simile ci sembra vadano le tecniche di nudging: «A nudge, as we will use the term, is any aspect of the choice architecture that alters people's behavior in a predictable way without forbidding any options or significantly changing their economic incentives. To count as a mere nudge, the intervention must be easy and cheap to avoid. Nudges are not mandates.» (Thaler e Sustein, 1989).

6 Il tema di Why are we wired that way? è peraltro affrontato da alcuni importanti studi sull'atteggiamento pro-cooperativo presente nella nostra specie. Si veda per questo il lavoro fondamentale di Bowles, Gintis (2011). 


\section{L'impatto recente della rivoluzione comportamentale sulle discipline manageriali}

A dispetto di una lunga tradizione di studi osservativi e sperimentali nel management ${ }^{7}$, il modo prevalente con il quale le evidenze della seconda rivoluzione comportamentale sono state applicate alle decisioni manageriali è stato di offrire una serie di avvertenze e cautele volte ad evitare al manager di cadere nelle stesse trappole decisionali descritte dall'economia comportamentale. Nella misura in cui molte fonti di bias sono sistematiche e prevedibili, queste linee guida comportamentali mettono in guardia il decisore dall'applicazione intuitiva e ingenua di strumenti o di approcci decisionali che rischiano di risultare in giudizi distorti o decisioni subottimali. La letteratura manageriale si è così arricchita di prontuari che evidenziano le trappole decisionali e propongono metodi per evitarle interamente entro una tradizione prescrittiva. L'incontro tra studiosi sperimentali del comportamento economico e studiosi di management è stato fruttuoso ed ha permesso di produrre liste di possibili errori e di suggerimenti per evitarli (Ariely 2010; Bazerman e Chugh 2006; Gino, 2013; Hammond et al., 1998). Nell'insegnamento del management, poi, il confronto con quelle evidenze sperimentali ha un effetto importante in quanto propone vie nuove per creare un "buon decisore": questo dovrà non solo servirsi di adeguati modelli, ma anche imparare che, per usarli in modo appropriato, è necessario esercitare un po' di violenza contro le nostre tendenze istintive, e correggere la direzione verso cui le naturali dinamiche sociali e organizzative ci condurrebbero. Alcuni esempi possono chiarire il modo di procedere che viene suggerito.

Nella ricerca delle informazioni rilevanti per una specifica decisione, spesso ci si dedica prevalentemente alla raccolta di evidenze che confermano ipotesi e modelli interpretativi del problema, mentre si trascurano le informazioni discordanti con il quadro che si è costruito (bias della conferma, cfr. per una rassegna Klayman e Ha, 1987). Essere consci della tendenza sistematica a trascurare questa seconda categoria di strategie di ricerca delle informazioni è il primo passo verso una presa di decisione più consapevole e informata.

Un altro esempio è quello di un bias decisionale molto conosciuto che va sotto il nome di arbitrary coherence (Ariely et al., 2003). Secondo questa evidenza il manager può essere indotto a sequenze di scelte derivate (in modo coerente) da un ancoraggio iniziale arbitrario. Ad esempio, l'allocazione dei budget alle divisioni procede spesso per aggiustamenti internamente coerenti che assumono come un dato incontestabile la situazione di partenza e producono aggiustamenti coerenti rispetto a quella che invece è una allocazione iniziale arbitraria. Volendo derivare delle implicazioni quindi, la letteratura manageriale indica che per evitare fenomeni di ancoraggio e di arbitrary coherence sarà necessario adottare un approccio

Per una ricostruzione storica sulla diffusione della prospettiva comportamentale all'interno degli studi manageriali si rinvia a Aronoff (1975). 
decisionale capace di mettere in discussione il riferimento iniziale, per riportarci a una situazione zero based.

Infine, così come conosciamo l'esistenza di bias nelle procedure di decisione, sappiamo anche che abbiamo molteplici problemi ad apprendere e a trasferire l'esperienza ad altri casi. Molti studi sperimentali mettono in luce la diversa salienza nei processi di apprendimento dei casi positivi rispetto a quelli negativi. Noi tutti tendiamo a dimenticare facilmente le cose che ci vanno male, e ad attribuirne l'esito non alle nostre scelte, ma a fattori estranei a noi. Viceversa ricordiamo facilmente le esperienze positive e ce ne attribuiamo il merito, poiché ci viene naturale minimizzare la dissonanza cognitiva che il ricordo di situazioni spiacevoli crea, in maniera del tutto analoga a quanto accade nel già visto bias della conferma (Harrison e March, 1984). La nostra memoria, insomma, è viziata nel senso di sedimentare in modo diverso esperienze positive e negative. Questo fenomeno è acuito da comportamenti organizzativi e sociali. Infatti, le organizzazioni tendono a promuovere i manager che hanno successo i quali diffondono il proprio repertorio dei casi, viziato verso le esperienze positive (Levinthal e March, 1993). Per far fronte a questo bias presente nei processi di apprendimento analogico, la memoria individuale può essere arricchita e corretta da procedure tese a esplorare in modo più oggettivo le esperienze svolte. Il ruolo degli osservatori esterni è altresì molto importante, perché aiuta a ottenere una rappresentazione più neutrale degli avvenimenti.

L'operazione di "correzione dei bias" ora descritta è però assai più discutibile di quella realizzata dall'economia per incorporare i risultati sperimentali in modelli prescrittivi. Il designer descritto da Roth interviene su una popolazione di agenti ingenui che prendono decisioni abbastanza semplici, assimilabili a quelle studiate sperimentalmente. Quando questa procedura si applica al management, occorre però tener conto almeno dell'esistenza di problemi di generalizzazione e problemi di coerenza logica.

Problemi di generalizzazione. I manager operano in contesti caratterizzati da una scarsa strutturazione dei problemi e da rilevanti elementi di ambiguità interpretativa. Spesso si è in presenza di decisori incerti sulle stesse conseguenze logiche di quanto conoscono. Essi subiscono una incertezza non limitata agli eventi che determinano il risultato delle proprie azioni (Radner, 1996). La domanda fondamentale è quindi: questi problemi decisionali sono riconducibili a quelli riprodotti sperimentalmente? Nei prontuari per la formazione di decisioni unbiased si ipotizza che sia possibile ricondurre il problema decisionale a una forma trattabile all'interno di una procedura di decisione razionale. Ma le deviazioni dalla razionalità che si osservano, saranno davvero tali, frutto cioè di un decisore impreparato e ingenuo? In realtà sappiamo ancora molto poco del ruolo dei bias in contesti articolati, e della loro interazione. Affermare che le deviazioni osservate in contesti semplici saranno riprodotte e amplificate in situazioni decisionali più articolate, e che quindi è importante correggere errori pervasivi indipendentemente dal contesto decisionale, rischia di produrre interpretazioni falsate degli eventi. Ad esempio, da anni la letteratura di natura manageriale sottolinea il fatto che nelle fusioni e acquisizioni, molto spesso, 
il controllo viene pagato troppo, ad un prezzo, insomma, non giustificato dai ritorni attesi. Nondimeno le fusioni e acquisizioni continuano ininterrotte. Di fronte a situazioni simili, il comportamentismo che abbiamo descritto in precedenza evidenzierebbe una molteplicità di fenomeni di irrazionalità: un fenomeno indicato, ad esempio, come overconfidence (Fischhoff et al., 1977), per il quale siamo disposti a credere di essere in grado di governare gli eventi in misura molto maggiore di quanto effettivamente possiamo fare. Scopriremmo probabilmente fenomeni di apprendimento self confirming, per il quale si attribuisce a se stessi l'effetto di una decisione andata a buon fine, mentre si biasima la sfortuna, o eventi esterni non controllati quando i risultati sono cattivi, con l'effetto di una analisi parziale e viziata delle esperienze trascorse. Tutti questi fenomeni, effettivamente, potrebbero essere presenti. Ma chiediamoci: è davvero possibile che decisori esperti, che hanno di fronte a sé anni e anni di letteratura ed esempi, siano così sciocchi da ripetere gli stessi errori? È ben vero che, per dirla con il titolo di un fortunato libro di Dan Ariely, siamo in presenza di "predictable irrationality", dalla quale nessuno è esente. E tuttavia non solo questi fenomeni sono noti da tempo, ma anche il libro di Dan Ariely (2010) non dovrebbe essere sfuggito almeno a qualcuno del team che si è occupato dell'operazione e che dovrebbe aver messo in guardia il vasto gruppo di analisti coinvolto dei rischi di un comportamento irrazionale. Non è il caso allora di analizzare più a fondo che cosa sia successo in quei casi e di immaginare che $i$ notevoli risultati dell'economia comportamentale possano tradursi in qualcosa di più di un elenco di caveat che accompagna il buon decisore?

Problemi di coerenza logica. Un modello di decisione manageriale deve necessariamente comprendere anche gli stessi manager: quelli che operano nella medesima impresa contribuendo a definirne le decisioni, e quelli che operano in altre imprese le cui decisioni interagiscono con le proprie. Non è chiaro quale schema debba essere adottato quando, nel problema decisionale, entrano altri decisori oltre al manager in questione. Come si comporterà la controparte che interagisce con noi (la quale a propria volta avrà sicuramente letto il libro di Dan Ariely)? Sarà un decisore razionale in grado di emendare le proprie "irrazionalità", $\mathrm{o}$ un manager improvvido che ancora non ha raffinato i processi decisionali e vive nell'incoscienza dei propri limiti?

\section{Una diversa prospettiva per un approccio comportamentale al management}

Mentre continuano a cumularsi in economia evidenze su come il comportamento decisionale umano non rispetti gli assunti e le previsioni dei modelli classici di scelta, molti autori (per esempio Gigerenzer, 2007; Kahneman e Tversky, 2000) sostengono che le «deviazioni sono il prodotto naturale dei processi cognitivi e suggeriscono dunque che gli errori che compiamo nelle decisioni e nel ragionamento sono costitutivi del modo di funzionare della mente umana» (Egidi, 2005). Vedere nelle procedure decisionali e nelle euristiche fast and frugal non irrazionalità, ma 
soluzioni intelligenti per muoversi rapidamente, con i nostri limiti cognitivi, in ambienti decisionali complessi, sembra dunque una strada interessante e promettente che ci riporta alla prima rivoluzione comportamentale e ai tentativi di impiegare osservazioni controllate per scoprire la natura delle procedure decisionali adottate da persone e organizzazioni caratterizzate ognuna da precisi vincoli strutturali, fisici o di disegno. Secondo una visione evoluzionistica (Bingham e Eisenhardt, 2011), i bias si sono preservati, nonostante diano in molti casi risultati peggiori di una procedura razionale, perché, dovendosi muovere rapidamente in un ambiente difficile, l'uomo ha sviluppato metodi per reagire prontamente a cose che non conosce. Le euristiche producono con rapidità soluzioni "abbastanza buone" (good enough) utilizzando procedure generali che non necessitano di una analisi accurata del contesto, ma che si avvalgono di informazioni locali. Certamente tali procedure si preservano e possono diventare disfunzionali quando, aumentando il controllo sull'ambiente, si continuano ad applicare le stesse euristiche a problemi controllabili. La questione centrale delle decisioni manageriali, in questa prospettiva, è data dalla necessità di raggiungere un bilanciamento fra accuratezza della procedura decisionale e complessità del problema. I termini di questo trade off, tuttavia, non sono definiti una volta per tutte, ma variano insieme alla capacità di intervenire sull'ambiente per renderlo più trattabile. Un caso eclatante di come tale trade-off sia cambiato nel tempo è costituito dalla sfida a scacchi che l'intelligenza artificiale ha lanciato ai grandi maestri del gioco. Essendo il gioco degli scacchi un problema di grande dimensione, difficile sotto il profilo computazionale, ma sostanzialmente definito, a partire dagli anni cinquanta si incominciò a pensare che un calcolatore avrebbe agevolmente potuto avere la meglio sull'uomo. Per questo, in una ricostruzione della vicenda, Herbert Simon e Jonathan Schaeffer ne parlano come della drosophilia dell'intelligenza artificiale (Simon e Schaeffer, 1992). Si contrastarono all'epoca due scuole di pensiero: l'una era l'approccio brute force, per il quale si doveva investire nelle capacità di calcolo delle macchine forzando la costruzione completa dell'albero del gioco (o almeno di segmenti rilevanti di questo). L'approccio alternativo indicava una via diversa consistente nello studio diretto del comportamento dei grandi maestri. È necessario considerare, tuttavia, che di fronte a un problema difficilmente trattabile (sia pure solo dal punto di vista dimensionale, come nel caso degli scacchi) entrambe le procedure di soluzione includono, se vogliamo chiamarli così, bias: da un lato quello derivante dall'uso di procedure di ricerca, dall'altro quello derivante dall'applicazione di un modello decisionale razionale in un contesto non completamente trattabile, in questo caso a motivo della limitata capacità di calcolo. Tra questi due bias esiste un trade off che può variare nel tempo. Se, quando Simon e Schaeffer scrivevano la loro rassegna, i due approcci si confrontavano sostanzialmente alla pari la situazione ora è cambiata, al punto che programmi per il calcolatore mettono ora non solo in crisi molti grandi maestri, ma anche campioni mondiali.

Ciò ci suggerisce che il significato delle euristiche, se esse siano un meccanismo efficace fast and frugal o se costituiscano "errori di cablaggio" della mente umana, è in certo modo mal posta. Tanto i modelli di razionalità astratta che le euristiche 
sviluppate dall'uomo devono essere "costruttivamente" percorribili e devono fare i conti con vincoli fisici e ambientali che, a loro volta, non sono dati ma possono cambiare, così come può cambiare la capacità di calcolo umana con lo sviluppo di macchine più potenti, o la capacità di immagazzinare e recuperare conoscenza in strutture organizzative diffuse in cui singoli individui si specializzano.

Queste considerazioni fanno pensare che un programma di ricerca comportamentale per il management non possa semplicemente mutuare metodi e risultati della seconda rivoluzione comportamentale che hanno avuto successo in economia. Il manager (come forse in misura ancora maggiore l'imprenditore) affronta problemi complessi, interagendo spesso con altri agenti altrettanto sofisticati. Egli inoltre è in grado di manipolare lo stesso problema decisionale, modificandone il grado di complessità. Per questo è opportuno sviluppare un appropriato programma di ricerca che tenga conto almeno di tre aspetti.

1. È necessario anzitutto adottare una prospettiva evoluzionistica, che tenga conto dei processi di apprendimento e selezione che affiancano l'uso delle euristiche e che sottendono ai bias. È ragionevole infatti pensare che in molti casi i nostri errori siano la conseguenza dell'impiego di procedure cognitive di successo, che hanno permesso all'uomo di ottenere vantaggi evolutivi nell'affrontare particolari situazioni decisionali difficili e mal formulate (Haselton et al., 2005). Tali procedure tuttavia possono contenere componenti che, in altri contesti, possono dar luogo a bias decisionali importanti per i quali la letteratura ha fornito una serie di correttivi puntuali. Ad esempio, in un processo di ricerca in un contesto poco conosciuto, può essere naturale partire da un punto iniziale casuale e valutare i miglioramenti o peggioramenti rispetto a questo, senza chiedersi se la nostra ricerca sia partita da un punto di vista corretto. Tale euristica di ricerca potrebbe essere corretta da una procedura di revisione degli ancoraggi, che agisce su orizzonti temporali diversi ed è attivata solo da eventi che producono risultati al di fuori di un certo intervallo di variazione. Tuttavia, sia i bias che le relative correzioni possono consolidarsi ed essere impiegati in modo inappropriato in singole decisioni, se si ignorano le condizioni contestuali per cui certe euristiche sono state selezionate poiché rispetto ad esse hanno mostrato la propria efficacia. Pertanto, se si vogliono correggere i processi spontanei di decisione, è importante non solo emendare i vizi, ma capirne il ruolo, le modalità di funzionamento e le condizioni di efficienza.

2. Il successo evolutivo delle euristiche va a propria volta valutato su un piano ecologico e di diversità. Ciò che può risultare funzionale potrebbe essere non la singola regola, ma la popolazione delle stesse che interagendo produce una ricerca che dà buoni risultati. Ad esempio, come mostrano Bernardo e Welch (2001), la presenza di soggetti overconfident può dare un vantaggio alla popolazione in cui questi sono presenti, perché preserva informazione e bilancia il comportamento di herding di altri soggetti che utilizzano l'informazione derivata dall'osservazione delle scelte altrui. Del pari, soggetti con comportamenti self confirming permettono di aumentare l'esplorazione di nuove soluzioni. Ancora, una letteratura sempre più consistente e che ora sembra 
trovare qualche conferma anche sul piano neurologico (Laureiro-Martínez et $a l ., 2010)$, indica che gli imprenditori utilizzano meccanismi cognitivi diversi da altri decisori sofisticati. Il successo evolutivo di una popolazione potrebbe essere data da una composizione adeguata di persone che ragionano al modo degli imprenditori e persone che seguono procedure decisionali più conservative. L'individuazione delle proprietà di decisori collettivi che adottano regole diverse dovrebbe essere una componente fondante della teoria comportamentale dell'organizzazione, in cui le decisioni sono il risultato dell'interazione tra persone con diverse conoscenze, competenze ed esperienze. Da questo punto di vista, ci sembrano esemplari gli studi di Scott Page (Hong e Page, 2004; Page, 2007) sull'effetto della diversità e sulla capacità di gruppi di agenti con limiti decisionali di avere performance decisionali superiori a gruppi di agenti esperti. Alcune domande di ricerca che qui possiamo sollevare riguardano quindi la mappatura della diversità (qual è e su quali aspetti si basa la diversità rilevante per la decisione) e su come beneficiare di decisori diversi.

3. Semplici euristiche risultano dalla necessità di affrontare problemi complessi in presenza di stretti limiti di alcune capacità cognitive, e permettono di ottenere, in particolari condizioni, risultati migliori dei modelli di scelta razionale (Gigerenzer et al., 1999; Payne et al., 1993). La natura del problema decisionale non è però data una volta per tutte. Il manager non è solo un decisore, egli bensì struttura il problema decisionale in modo da ridurne la complessità. La capacità di trattare problemi complessi dipende sia dalle risorse e dalle tecnologie a disposizione, sia dalle capacità cognitive e di rappresentazione del decisore, che modificano la trattabilità del problema. L'uomo si dota di strategie che permettono di semplificare l'ambiente e di ridurre pertanto il carico cognitivo e la flessibilità richiesti per muoversi in un ambiente difficile. Se, per camminare in un ambiente naturale, abbiamo bisogno di articolazioni capaci di adattarsi a molteplici movimenti, quelle strutture diventano meno efficienti quando un intervento sull'ambiente (con la costruzione di strade e ponti) lo rende meno complesso e maggiormente prevedibile. Allo stesso modo anche l'ambiente decisionale può essere organizzato e semplificato, in modo da ridurre le interazioni, per esempio, decomponendolo in sottosistemi indipendenti, o specializzando i decisori per agire in sottosistemi specifici. Regole e convenzioni possono poi ridurre lo spazio dei comportamenti ammessi. Anche i vincoli fisici interni al decisore possono modificarsi: lo sviluppo di algoritmi migliori può ridurre la necessità di calcolo e la nostra limitata memoria può essere ampliata dall'uso della parola scritta o dai calcolatori. Il trade-off tra euristiche e calcolo razionale può così cambiare, sia per l'aumentata capacità di ridurre la complessità ambientale, sia per lo sviluppo di tecniche che accrescono conoscenza, memoria e velocità di analisi dei problemi. Se le procedure decisionali da adottare non sono iscritte nel problema, ma dipendono dalle risorse mentali e tecniche che danno forma alla rappresentazione del problema, uno dei temi fondamentali di ricerca diventa quello di riconoscere i contesti nei quali applicare diverse procedure decisionali, e come ottenerne una 
rappresentazione il più possibile adeguata ai mezzi a disposizione. Una delle lezioni fondamentali della prima rivoluzione comportamentale è stata di distinguere decisioni programmate e no (Simon, 1988), e l'evidenza conseguente della non unicità delle tecniche decisionali. Del pari, il famoso apologo di Tempus e Hora (Simon, 1962) illustra bene come sia possibile modificare le proprietà dei problemi attraverso diverse rappresentazioni.

\section{Conclusioni}

Mentre la prima rivoluzione comportamentale per il suo messaggio originario è stata recepita maggiormente nell'ambito degli studi organizzativi e manageriali, la seconda ha trovato un terreno fertile nell'economia. I risultati di questa seconda ondata di studi, mirante ad identificare violazioni delle condizioni e delle procedure di decisione tipiche della teoria standard agli studi manageriali, hanno trovato applicazione al management attraverso un insieme di prescrizioni che dovrebbero insegnare al decisore ad evitare le trappole insite nei processi decisionali e derivanti dalla psicologia umana.

In questo articolo abbiamo mostrato come questo tipo di applicazioni delle scoperte dell'economia comportamentale alle decisioni manageriali presenti severi limiti. Abbiamo mostrato inoltre come quei risultati possano essere interessanti ed utili se integrati in una prospettiva che riprende lo spirito del programma di ricerca della prima rivoluzione comportamentale, oggi inspiegabilmente relegata al margine negli studi manageriali, largamente dominati da temi tipici della seconda rivoluzione.

Abbiamo per questo tracciato le caratteristiche di un tale programma, che a nostro giudizio dovrebbe: 1. avere un carattere evolutivo, inserendo l'analisi dei processi decisionali in un contesto di apprendimento e selezione; 2 . avere un carattere ecologico, cioè considerare l'effetto di popolazioni di decisori dotati di regole diverse; 3 . approfondire la relazione tra modelli decisionali e natura dei problemi, comprendendo in questo anche la possibilità di usare risorse tecniche e cognitive per modificare la natura dei problemi affrontati.

Nell'articolo che conclude un recente numero speciale dello Strategic Management Journal dedicato agli aspetti comportamentali negli studi strategici, Daniel Levinthal (2011) riprendendo il dibattito tra i due approcci comportamentali, scrive:

«The choice is not between whether we should act in a God-like manner, like mortals. We are mortals. Given this second-best setting, the question is how to act with intelligence in strategic contexts. Business strategy is not a 'tic-tac-toe' environment in which explicit optimum of the real strategic context can be derived. Further, one can act with intelligence even in the absence of deliberative reasoning. Adaptive learning, selection mechanisms and imitative processes are all alternative bases of intelligence. Nonetheless, deliberative 
reasoning and analysis is an important mechanism. But such process

do not live wholly apart from behavioral mechanisms.»

Scoprire come produrre un'azione intelligente in contesti difficili costituisce la vera sfida attuale degli studi comportamentali. Nella ricerca e nella pratica manageriale questa sfida è in gran parte ancora da cogliere.

\section{Bibliografia}

AKERLOF G.A., KRANTON R.E. (2010), Identity economics: How our identities shape our work, wages, and well-being, Princeton University Press, Princeton, NJ.

ARIELY D., LOEWENSTEIN G., PRELEC D. (2003), “'Coherent arbitrariness': Stable demand curves without stable preferences", The Quarterly Journal of Economics, vol. 118 , n. 1, pp. 73-106.

ARIELY D. (2010), Predictably irrational, (rev. ed.), Harper Perennial, London.

AUGIER M., MARCH J.G. (2011), The roots, rituals and rhetorics of change: North American business schools after the Second World War, Stanford University Press, Stanford, CA.

BACCARANI C. (2010), "Complessità e intelligenza manageriale", Sinergie, n. 81, pp. 97111.

BAZERMAN M.H., CHUGH D. (2006), "Decisions without blinders", Harvard Business Review, vol. 84, n.1, pp. 88-97.

BAZERMAN M.H., MOORE D.A. (2009), Judgment in managerial decision making. (settima ed.), John Wiley and Sons, Hoboken, NJ.

BELL D.E., RAIFFA H., TVERSKY A. (1988), "Descriptive, normative, and prescriptive interactions in decision making", in Bell D.E., Raiffa H., Tversky A. (a cura di), Decision making. Decriptive, normative and prescriptive interactions, Cambridge University Press, Cambridge, pp. 9-30.

BERNARDO A.E., WELCH I. (2001), "On the evolution of overconfidence and entrepreneurs", Journal of Economics and Management Strategy, vol. 10, n. 3, pp. 301-330.

BINGHAM C.B., EISENHARDT K.M. (2011), "Rational heuristics: the 'simple rules' that strategists learn from process experience", Strategic Management Journal, vol. 32, n. 13, pp. 1437-1464.

BOWLES S., GINTIS H. (2011), A cooperative species: Human reciprocity and its evolution, Princeton University Press, Princeton, NJ.

BRUNETTI F. (2010), "Di cosa parliamo quando parliamo di 'scienze manageriali'?", Sinergie, n. 86, pp. 69-89.

CAMERER C.F. (2003), Behavioral game theory: Experiments in strategic interaction, Russell Sage Foundation \& Princeton University Press, New York \& Princeton, NJ.

CAMERER C.F., LOEWENSTEIN G. (2004), "Behavioral economics: Past, present, future", in Camerer C.F., Loewenstein G., Rabin M. (a cura di), Advances in behavioral economics, Princeton University Press, Princeton, Russell Sage Foundation ed., NJ, pp. 3-51.

CHARNESS G., RABIN M. (2002), "Understanding social preferences with simple tests", Quarterly Journal of Economics, vol. 117, n. 3, pp. 817-869. 
CHOPLIN J.M., HUMMEL J.E. (2005), “Comparison-induced decoy effects”, Memory \& Cognition, vol. 33, n. 2, pp. 332-343.

COHEN M.D., MARCH J.G., OLSEN J.P. (1972), “A garbage can model of organizational choice", Administrative Science Quarterly, vol. 17, n. 1, pp. 1-25.

CYERT R.M., MARCH J.G. (1963), A behavioral theory of the firm, Prentice-Hall, Englewood Cliffs, NJ.

DELLA VIGNA S. (2009), "Psychology and economics: Evidence from the field", Journal of Economic Literature, vol. 47, n. 2, pp. 315-372.

DEVETAG G., LEGRENZI P., WARGLIEN M. (1997), “Come le alternative inefficienti influenzano le nostre decisioni”, Sistemi Intelligenti, vol. 9, n. 3, pp. 377-398.

EGIDI M. (2005), "Errori e fallibilità", Networks, n. 5, pp. 102-115.

FEHR E., GÄCHTER S. (2000), "Fairness and retaliation: The economics of reciprocity" The Journal of Economic Perspectives, vol. 14, n. 3, pp. 159-181.

FEHR E., SCHMIDT K. M. (1999), "A theory of fairness, competition, and cooperation", Quarterly Journal of Economics, vol. 114, n. 3, pp. 817-868.

FEHR E., CAMERER C.F. (2007), "Social neuroeconomics: the neural circuitry of social preferences", Trends in Cognitive Sciences, vol. 11, n. 10, pp. 419-427.

FISCHHOFF B., SLOVIC P, LICHTENSTEIN S. (1977), "Knowing with certainty: The appropriateness of extreme confidence", Journal of Experimental Psychology: Human Perception and Performance, vol. 3, n. 4, pp. 552-564.

GIGERENZER G., TODD P.M., ABC RESEARCH GROUP, (a cura di) (1999), Simple heuristics that make us smart, Oxford University Press, New York, NY.

GIGERENZER G. (2007), Gut feelings: The intelligence of the unconscious, Viking Press, New York, NY.

GINO F. (2013), La scelta giusta, Sperling Kupfer, Milano.

GNEEZY U., RUSTICHINI A. (2000), "Pay enough or don't pay at all", The Quarterly Journal of Economics, vol. 115, n. 3, pp. 791-810.

HALL R.L., HITCH C.J. (1939), "Price theory and business behaviour", Oxford Economic Papers, n. 2, pp. 12-45.

HAMMOND J.S., KEENEY R.L., RAIFFA H. (1998), "The hidden traps in decision making" Harvard Business Review, vol. 76, n. 5, pp. 47-58.

HARRISON J.R., MARCH J.G. (1984), "Decision making and postdecision surprises", Administrative Science Quarterly, vol. 29, n. 1, pp. 26-42.

HASELTON M.G., NETTLE D., ANDREWS P.W. (2005). "The evolution of cognitive bias", in Buss D.M. (a cura di), The handbook of evolutionary psychology (pp. 724746), John Wiley, Hoboken, NJ.

HARROD R.F. (1939), "An essay in dynamic theory", The Economic Journal, vol. 49, n. 193, pp. 14-33.

HENRICH J., BOYD R., BOWLES S., CAMERER C., FEHR E., GINTIS H., MCELREATH R. (2001), "Cooperation, reciprocity and punishment in fifteen small-scale societies", American Economic Review, vol. 91, n. 2, pp. 73-78.

HONG L., PAGE S.E. (2004), "Groups of diverse problem solvers can outperform groups of high-ability problem solvers", Proceedings of the National Academy of Sciences of the United States of America, vol. 101, n. 46, pp. 16385-16389.

HOSSEINI H. (2003), "The Arrival of Behavioral Economics: From Michigan, or the Carnegie School in the 1950s and the early 1960s?", Journal of Socio-Economics, vol. 32, n. 4, 391-409. 
HUBER J., PAYNE J.W., PUTO C. (1982), “Adding Asymmetrically Dominated Alternatives: Violations of Regularity and the Similarity Hypothesis", Journal of Consumer Research, vol. 9, n.1, pp. 90-98.

KAHNEMAN D., TVERSKY A. (1979), "Prospect theory: An analysis of decision under risk", Econometrica, vol. 47, n. 2, pp. 263-291.

KAHNEMAN D., TVERSKY A., a cura di (2000), Choices, values, and frames, Cambridge University Press, Cambridge, MA.

KLAYMAN J., HA Y.W. (1987), "Confirmation, disconfirmation, and information in hypothesis testing", Psychological Review, vol. 94, n. 2, pp. 211-228.

LAUREIRO-MARTÍNEZ D., BRUSONI S., ZOLLO M. (2010), "The neuroscientific foundations of the exploration-exploitation dilemma", Journal of Neuroscience, Psychology, and Economics, vol. 3, n. 2, pp. 95-115.

LEVINTHAL D.A., MARCH J.G. (1993), “The myopia of learning”, Strategic Management Journal, vol. 14, n. S2, pp. 95-112.

LEVINTHAL D.A. (2011), "A behavioral approach to strategy - what's the alternative?", Strategic Management Journal, vol. 32, n. 13, pp. 1517-1523.

LINDMAN H.R. (1971), "Inconsistent Preferences among Gambles", Journal of Experimental Psychology, n. 89, vol. 2, pp. 390-397.

MARCH J.G. (1994), A primer on decision making: How decisions happen, Simon and Schuster, New York, NY.

MARCH J.G., SIMON H.A. (1993), Organizations, Blackwell Publishers, Cambridge, MA.

MAYO E. (1933), The human problems of an industrial civilization, MacMillan, New York, NY.

MAYO E. (1945), The social problems of an industrial civilization, Division of Research, Graduate School of Business Administration, Harvard University, Boston, MA.

PAGE S.E. (2007), The difference: How the power of diversity creates better groups, firms, schools, and societies, Princeton University Press, Princeton, NJ.

PAYNE J.W., BETTMAN J.R., JOHNSON E.J. (1993), The Adaptive Decision Maker, Cambridge University Press, Cambridge, MA.

PETTIBONE J.C., WEDELL D.H. (2007), "Testing alternative explanations of phantom decoy effects", Journal of Behavioral Decision Making, vol. 20, n. 3, pp. 323-341.

RADNER R. (1996), "Bounded rationality, indeterminacy, and the theory of the firm", The Economic Journal, vol. 106, n. 438, pp. 1360-1373.

ROSSI A. (2013), Le decisioni manageriali. Modelli teorici ed evidenze empiriche, Franco Angeli, Milano.

ROTH A.E. (2002), "The economist as engineer: Game theory, experimentation, and computation as tools for design economics", Econometrica, vol. 70, n.4, pp. 13411378 .

SHAPIRA Z., (a cura di) (1997), Organizational decision making, Cambridge University Press, Cambridge, UK.

SIMON H.A. (1947), Administrative behavior, The Free Press, New York, NY.

SIMON H.A. (1955), "A behavioral model of rational choice", The Quarterly Journal of Economics, vol. 69, n. 1, pp. 99-118.

SIMON H.A. (1962), "The architecture of complexity", Proceedings of the American Philosophical Society, vol. 106, n. 6, pp. 467-482.

SIMON H.A. (1988), The new science of management decision. Englewood Cliffs, NJ, Prentice Hall. (trad. it.) Informatica, direzione aziendale e organizzazione del lavoro. La nuova scienza delle decisioni manageriali, Franco Angeli, Milano, 1988 (la prima edizione inglese è del 1960). 
SIMON H.A. (1987), "Bounded rationality", The new Palgrave. A dictionary of economics, Macmillan, London, pp. 266-268

SIMON H.A. (1996), The sciences of the artificial, (terza ed.), Macmillan, New York, NY. (la prima edizione è del 1947).

SIMON H.A., SCHAEFFER J. (a cura di) (1992), The game of chess, Elsevier, New York, NY.

SIMONSON I. (1989), "Choice based on reasons: The case of attraction and compromise effects", Journal of Consumer Research, vol. 16, n. 2, pp. 158-174.

SLOVIC P., LICHTENSTEIN S. (1968), "The Relative Importance of Probabilities and Payoffs in Risk Taking”, Journal of Experimental Psychology, n. 78, vol. 3, pt. 2, pp. $1-18$.

THALER R.H., SUSTER C.R. (2008), Nudge: improving decisions about health, wealth, and happiness, Yale University Press, New Haven, CT.

TVERSKY A. (1972), "Elimination by aspects: A theory of choice”, Psychological Review, vol. 79, n.4, pp. 281-299.

TVERSKY A., KAHNEMAN D. (1974), "Judgment under uncertainty: Heuristics and biases", Science, vol. 185, n. 4157, pp. 1124-1131.

TVERSKY A., KAHNEMAN D. (1981), "The framing of decisions and the psychology of choice", Science, vol. 211, n. 4481, pp. 453-458. 